\title{
Design of farmland information acquisition and transmission system Based on ZigBee wireless sensor network
}

\author{
Zheng Xu, Zhixing Shi, Yunfei Zhang, Yan Zhang \\ College of Information Science and Technology, Agricultural University of Hebei, Baoding, Hebei 071001
}

\begin{abstract}
In order to improve the network of agricultural management and intelligent level, reduce the workload of farmland management, this article completed the farmland information acquisition and transmission system based on ZigBee wireless sensor network design, focused on the systems architecture, hardware design and part of software design. The system can quickly, reliably remote acquisition and transmission of field information, is of great significance to the development of precision agriculture.

Index Terms - ZigBee; wireless sensor network; farmland information acquisition; precision agricultural
\end{abstract}

\section{Introduction}

Current agricultural information acquisition and transmission system is achieved through the wired communication technology design, but there are many drawbacks: First, although the wired communication technology equipment is easy to operate, and can block the outside interference, the farmland environment is very harsh, so the cable is easily eroded, especially in the rain season. Therefore, the application of the wired communication technology will lead to reduced precision rate of the farmland information acquisition and transmission, and bring inconvenience to the agriculture production; Secondly, most of the farmland information acquisition is made in a wide area. In the practical application of the agricultural production, in order to achieve the effective and comprehensive farmland acquisition, the sensor nodes should be placed in the monitoring area densely. However, it will cause the cable spread all over the monitoring area, and affect the growth of the crops. Moreover, the flexibility of entire system is low, and it is hard to extend the other functions. It also needs a lot of manpower and resources to maintain and replace the cable.

ZigBee is a new wireless network technology. It is shortdistance, low-power, low-cost, and low-speed, and it mainly used in short-distance wireless acquisition [1]. The wireless sensor network refers to a wireless network that consists of a large number of sensor nodes which is deployed in the detection region [2]. The wireless sensor network [3] is a selforganized distributed network, and composed of a large number of tiny sensor nodes with wireless communication capacity and computing power. It can monitor, perceive and collect the various environment or the information parameters of the detection objects, and it also has the abilities of automatic control, remote monitoring, and data computation.

The farmland information acquisition and transmission system aims at the demand of the current agricultural environment online monitoring. It is designed based on $\mathrm{ZigBee}$ wireless sensor network. It can make remote acquisition and transmission for the temperature, humidity, light intensity and other factors that affect the growth of crops in the monitored area efficiently and reliably. Therefore, it will improve the network and intelligent level of the agricultural management, and reduce the administrative workload of the farmland. It has great significance for agricultural production.

\section{Farmland Information Acquisition and Transmission System}

ZigBee farmland information acquisition and transmission system mainly includes server, remote client, GPRS module, ZigBee coordinator, ZigBee node and sensor node. The system architecture is shown in Fig. 1.

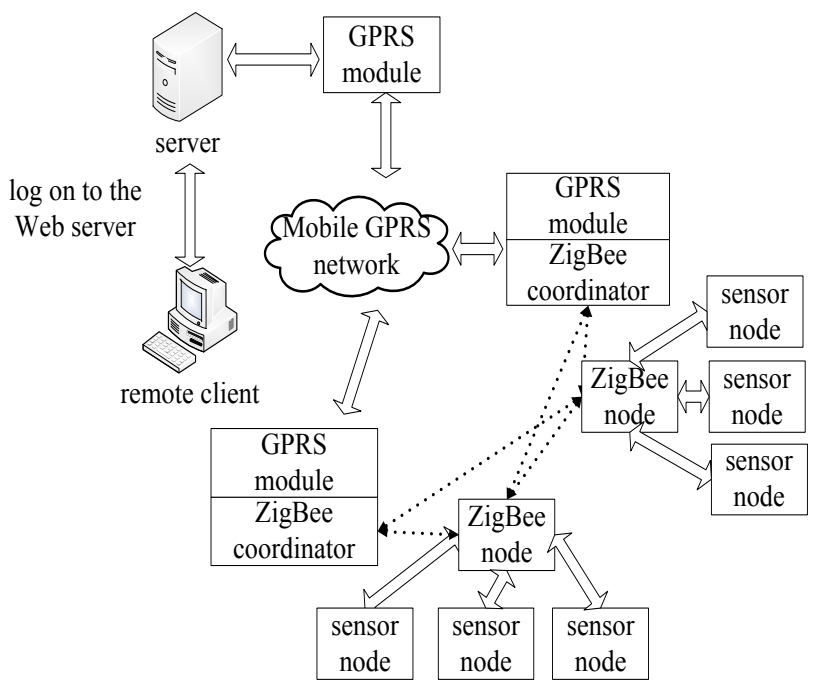

Fig.1 System architecture

The sensor node is a key part of the system, and is also the key point of the design. The nodes are placed in the farm-land monitored area randomly, and form a wireless sensor net-work rapidly by self-organization. Each sensor node serves as both collector and sender and information router within the sensor network [4-5]. In the whole system, the sensor nodes are mainly responsible for the acquisition of temperature, humidity, light degrees and other information of the farmland, 
and ZigBee nodes are responsible for the acquisition of the data information of the sensor nodes. The collected data information is sent to ZigBee coordinator through ZigBee wireless communication technology, then sent to the server through GPRS network, thus it is convenient for the remote client to login server, and to monitor the farmland environment in real time.

\section{Hardware Design of the System}

\section{A. Design of Wireless Sensor Network Node}

The design of wireless sensor network node is the core of the hardware design in the whole system. It is placed in the monitored area directly, and is responsible for the acquisition, process and transmission of the information. The design of wireless sensor network node must satisfy the special requirements of the specific application, such as miniaturization, low cost, low power, providing appropriate sensor for the node, necessary memory resources and appropriate communication equipment [6]. Wireless sensor network node consists of sensor module, microprocessor module, wireless communication module and power supply module. The structure of node is shown in Fig. 2.

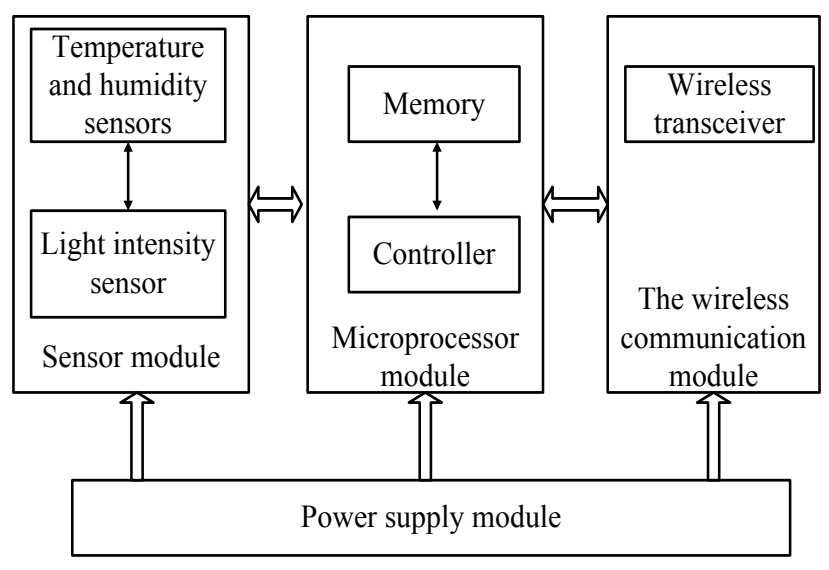

Fig.2 The structure of node

\section{B. Sensor Module}

The farmland information acquisition and transmission system collects the humidity, temperature and light intensity data information of the farmland. Due to the monitored area in the farmland and the conditions are relatively poor, there are many uncontrollable factors. Therefore, it is needed to consider the various factors when selects suitable sensor. The sensor should have excellent anti-interference, and be waterproof and heat resisting. It also should be small in size and easy to integrate, and low power to extend the service life. In conclusion, after analysis and comparison of various sensors, the SHT10 temperature and humidity sensor and the TSL2561 light intensity sensor are selected to measure the temperature, humidity and light intensity of the farmland environment respectively.

\section{The Wireless Communication Module}

In the test environment, a large number of nodes were placed in the monitored area randomly. Anti-interference ability and energy consumption should be given full consideration when select the chip. The chip should satisfy the signal processing requirements while resist interference and reduce the system energy consumption as far as possible. Taking all these factors into consideration, CC2430 form TI Corporation is selected as wireless transceiver chip of radiofrequency circuit among many wireless RF chips. It is mainly because the chip has super anti-interference ability and extremely low power consumption. It is a high cost-effective RF chip.

\section{Microprocessor Module}

The microprocessor module is the core part of the wireless sensor network node. Its main tasks include: First, it receives data that sends by the sensor node, and processes data, then sends them through the communication module; Second, it reads data and control information that receives by the communication module, operates data processing, and controls the other modules of the hardware platform; Third, it processes communication protocol, and completes MAC and routing protocol process in the wireless sensor network communication. In the choice of the microprocessor, there are various factors need to be considered, such as storage, process, peripheral interface, power consumption and so on. ATmegal6 from Atmel Corporation is selected. ATmegal6 is a high performance and low power consumption microprocessor. It has advanced instruction set and clock cycle instruction execution time. It also has maximum data throughput, and eases the contradictions between consumption and processing speed to a large extent.

\section{E. Power Supply Module}

The power supply module is the energy source of the wireless sensor network. The power technology decides the working hours of the network and the operating costs of the system. Furthermore, wireless sensor network is generally placed in the harsh environment or unattended areas. Some nodes are required to have mobility. Therefore, the nodes must be battery-powered. The power requirements of each module in the system are relatively low, and the voltage of $2.4 \mathrm{~V}$ to $3.6 \mathrm{~V}$ can make all modules work properly, thus two AA batteries can be used as a power source. Please remember to change the batteries regularly in order to make the wireless sensor network can be used normally.

\section{Software Design of the System}

\section{A. Software Design of Sensor Node}

Sensor nodes can collect and filtration farmland data actively, but also can receive and execute the coordinator instruction. The users can set the sensor acquisition time period according to the need. Procedure flow of sensor node is shown in Fig. 3. 


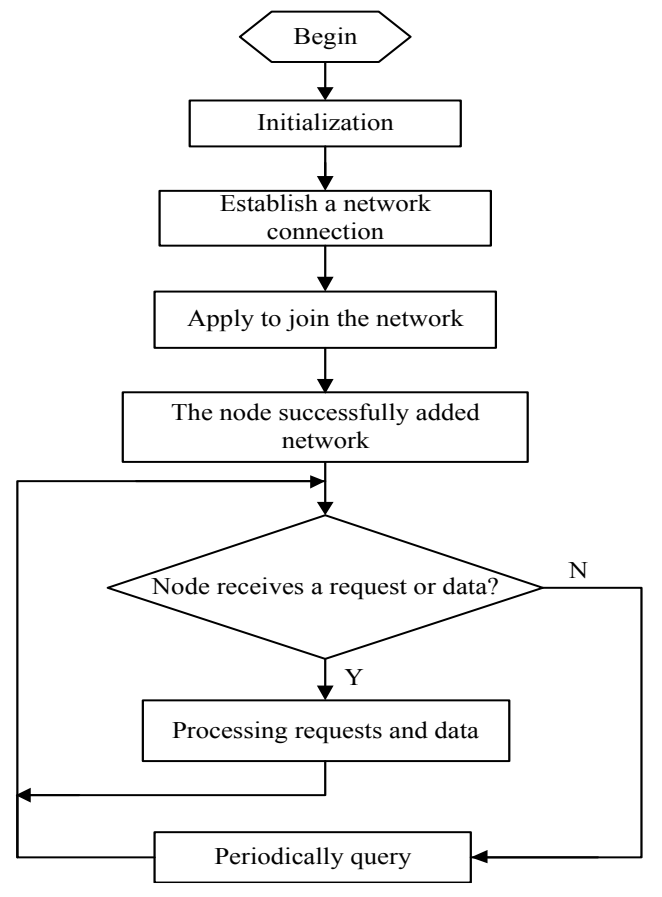

Fig.3 Procedure flow of sensor node

\section{B. Software Design of Coordinator Node}

Coordinator is the central node of the entire wireless network in ZigBee. It begins to work first. The main task of coordinator is to organize ZigBee network, allow the sensor nodes to join the network, bind the sensor nodes and send the collected data to the server. The procedure flow of coordinator is shown in Fig. 4.

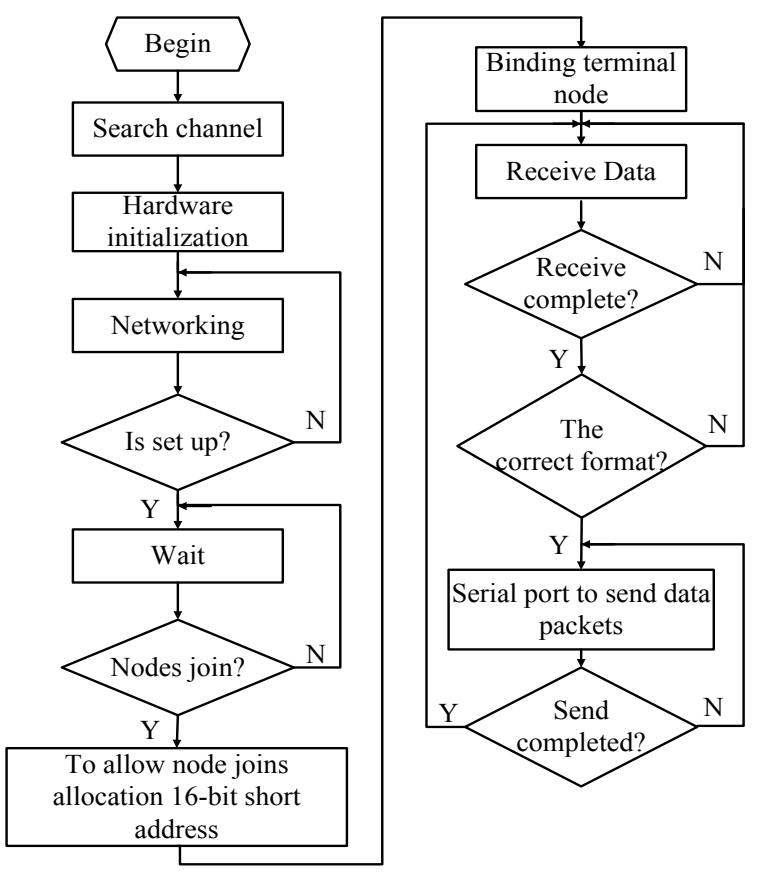

Fig.4 Procedure flow of coordinator

\section{1) Coordinator Network Construction}

Application layer initiates the network request to coordinator of network major node. The wireless sensor network is constructed after the request is received. The equipments that do not join the other network only have ZigBee FFD equipment capacity [7] can construct the new network. The coordinator network construction processes is shown in Fig. 5, description: In Fig. 5 and Fig. 6, APL, NWK and MAC denote the application layer, a network layer and a dielectric layer.

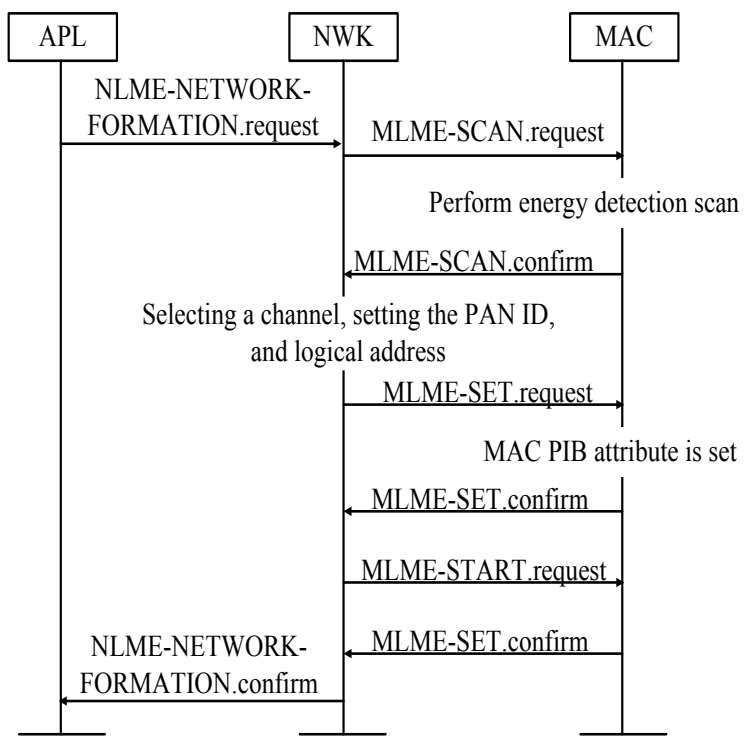

Fig.5 Coordinator network construction processes

\section{2) Coordinator allows devices to join the network}

ZigBee coordinator is in a waiting state after the coordinator constructs the network successfully. The coordinator will process the signal and allow the correspond-ing devices to join the network when detect the signal that requests to join the network. It is shown in Fig. 6.

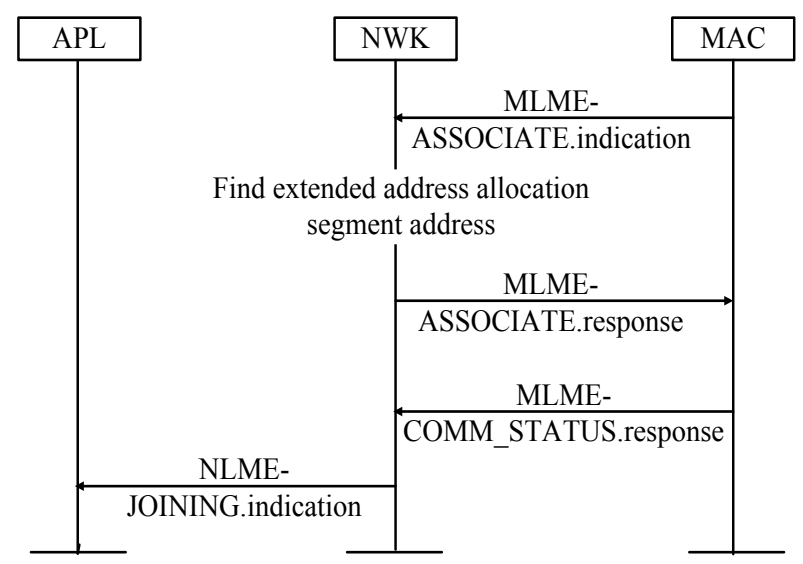

Fig.6 Allows devices to join the network 


\section{Conclusion}

The thesis applies wireless sensor network to acquisition and transmission of farmland information. It designs farmland information acquisition and transmission system bases on ZigBee wireless sensor network, and gives the overall design of the system, the hardware design of wireless sensor network node and part of software design processes. The system has low cost, low power, and flexible networking wireless sensor network technology, and avoids inconvenience of arrangement of wire. Introducing ZigBee technology into farmland information acquisition and transmission system is of great significance to the development of precision agriculture.

\section{References}

[1] Wenzhong Li, Zhaoyu Duan. ZigBee2006 wireless network with wireless positioning combat [M].Beijing: Beijing University of Aeronautics \& Astronautics Press, January 2008.

[2] Shu Wang, Yujie Yan, Fuping Hu. The theory and applications of wireless sensor networks[M].Beijing: Beijing University of Aeronautics and Astronautics Press, July 2007.

[3] Stankovic J.A, Abdelzaher T.F, Chenyang Lu. Real-time communication and coordination in embedded sensor networks $[\mathrm{P}]$. Proceedings of the IEEE, pp.1002-1022, July 2003.

[4] Jian Lu. Greenhouse wireless sensor networks based on the ZigBee protocol design [D]. Yangling: Northwest A \& F University, May 2010.

[5] Feng Gao. Research on the Automatic Monitoring System Based on Wireless Sensor Networks for Facility Agriculture Environment [M].Hangzhou: Zhejiang University of Technology. April 2009.

[6] Limin Sun, Jianzhong Li. Wireless sensor networks. Beijing: Tsinghua University Press. May 2005.

[7] Xiangming Wang. Research and Implementation of the ZigBee protocol network layer[J]. Application of Electronic Technique. pp.129-132, November 2006. 